\title{
Spin-Polarized Scanning Tunnelling Microscopy of Ultrathin Films
}

\author{
A. Kukunin, J. Prokop and H.J. Elmers \\ Institut für Physik, Johannes Gutenberg-Universität Mainz \\ Staudingerweg 7, 55099 Mainz, Germany
}

\begin{abstract}
Using low temperature spin-polarized scanning tunneling microscopy we have studied the morphology and magnetic properties of ultrathin $\mathrm{Fe}(0.5 \mathrm{ML}) \mathrm{Au}(0.5 \mathrm{ML})$ nanowires prepared on a Mo(110) single crystal. The Fe nanostripes grown by step flow on a Mo(110) at $700 \mathrm{~K}$ were covered by $\mathrm{Au}$ at RT, and subsequently annealed at $700 \mathrm{~K}$. Differences in the morphology of $\mathrm{Au}$ on $\mathrm{Fe}(110) / \mathrm{Mo}(110)$ and clean $\mathrm{Mo}(110)$ surfaces are observed and discussed. After annealing, the Mo is covered by a homogeneous FeAu ML alloy with several iron enriched islands which reveal an out-of-plane magnetic contrast. Direct lateral exchange coupling has been observed for these islands.
\end{abstract}

PACS numbers: 75.75.+a, 68.37.Ef, 81.15.-z

\section{Introduction}

Spin-polarized scanning tunneling microscopy (SP-STM) has become a powerful experimental method enabling studies of micromagnetism with an ultimate atomic resolution [1-3]. Spin-resolved tunneling experiments are very important for spintronics [4], where spin transport electronics is applied to the information media devices. We concentrate our attention on magnetic metallic nanostructures, an alternative for future spintronics applications $[4,5]$. Spin sensitive transport phenomena in two-dimensional ferromagnetic alloys are especially interesting, because these systems reveal different magnetic properties than in the bulk [5], and by the proper choice of substrate and preparation conditions, one can tailor magnetic properties of these systems, enabling spin engineering.

In this work we present results of low temperature SP-STM studies of the morphology and magnetic properties of ultrathin $\mathrm{FeAu}$ nanowires grown on a Mo(110) single crystal using molecular beam epitaxy (MBE). In general, the preparation of two-dimensional alloyed nanostructures involves different processes related to the MBE: preferential nucleation at specific sites of the substrate, anisotropic diffusion, and site exchange between the deposited atoms and the 
atoms at the surface [6], making the control of the local stoichiometry difficult. In addition, the $\mathrm{Fe} / \mathrm{Au}$ system is known for strong self-surfactant effects $[7,8]$. From another point of view, the $\mathrm{Fe} / \mathrm{Au}(100)$ system has attracted much attention in previous years due to its unique magnetic properties, which include ferromagnetism at the monolayer level [9], quantum well states [10], oscillatory magnetoresistance [11], and oscillatory magnetic coupling $[12,13]$. However, the structure, morphology, and magnetic properties of the two-dimensional FeAu alloys are unknown.

\section{Experimental details}

Experiments were performed in a UHV system (pressure $<1 \times 10^{-10}$ mbar) equipped with an evaporation system for MBE, sample characterization facilities and a low-temperature scanning tunneling microscope (LT-STM, Omicron NanoTechnology $\mathrm{GmbH})$. The Mo(110) single crystal was cleaned using the standard procedure including alternating flashing $(T \approx 2200 \mathrm{~K})$ in UHV and annealing $(T \approx 1400 \mathrm{~K})$ in an oxygen atmosphere $\left(p \approx 5 \times 10^{-8}\right.$ mbar $)$. The Fe nanostripes were prepared by step flow growth onto the Mo(110) substrate kept at $700 \mathrm{~K}$, and subsequently covered by Au at RT. Some samples were again annealed at $700 \mathrm{~K}$ for $6 \mathrm{~min}$. The STM measurements were performed at $5 \mathrm{~K}$. We use commercial tungsten tips which were flashed at $2200 \mathrm{~K}$, and subsequently covered by $10 \mathrm{ML}$ thick $\mathrm{Au}$ and Co layers at RT. In-plane and out-of-plane sensitive tips were obtained for different thicknesses of the Co coverage on the tip [14,15]. STM images were taken in a constant-current mode with the stabilized current of $1.5 \mathrm{nA}$ and gap voltage of $0.3 \mathrm{~V}$. Differential conductance $(\mathrm{d} I / \mathrm{d} U)$ maps were recorded simultaneously with the topography. The $\mathrm{d} I / \mathrm{d} U$ signal was measured by a lock-in technique applying a small modulation voltage of frequency $7 \mathrm{kHz}$ and $30 \mathrm{mV}$ amplitude.

\section{Results and conclusions}

Figure 1a shows the topographic STM image $\left(500 \times 500 \mathrm{~nm}^{2}\right)$ of a 0.5 pseudomorphic monolayer (ps-ML) Fe deposited onto the Mo(110) single crystal at $700 \mathrm{~K}$, and subsequently covered by $0.5 \mathrm{ML}$ Au at RT. For better understanding of this figure, we present the topography (Fig. 1b) and conductivity map (Fig. 1d) of the uncovered $\mathrm{Fe} / \mathrm{Mo}(110)$ surface. In Fig. 1b we see monoatomic Mo terraces which are decorated with the regular narrow Fe nanostripes grown by step flow growth at the Mo step edges. The positions of the Fe atoms on the Mo(110) surface can be better distinguished on the $\mathrm{d} I / \mathrm{d} U$ map (Fig. 1d) because the spin-averaged $\mathrm{d} I / \mathrm{d} U$ signal is different for the Fe and Mo surfaces [3]. Moreover, the Fe nanowires occasionally show two different colors on the $\mathrm{d} I / \mathrm{d} U$ map, although the spin-averaged conductance signals should be the same for these regions. This contrast has a magnetic origin, and results from spin sensitive tunneling between perpendicularly magnetized (either "up" or "down") Fe nanostripes [16] and the $\mathrm{W} / \mathrm{Au} / \mathrm{Co}$ tip with an out-of-plane magnetic sensitivity [15]. 


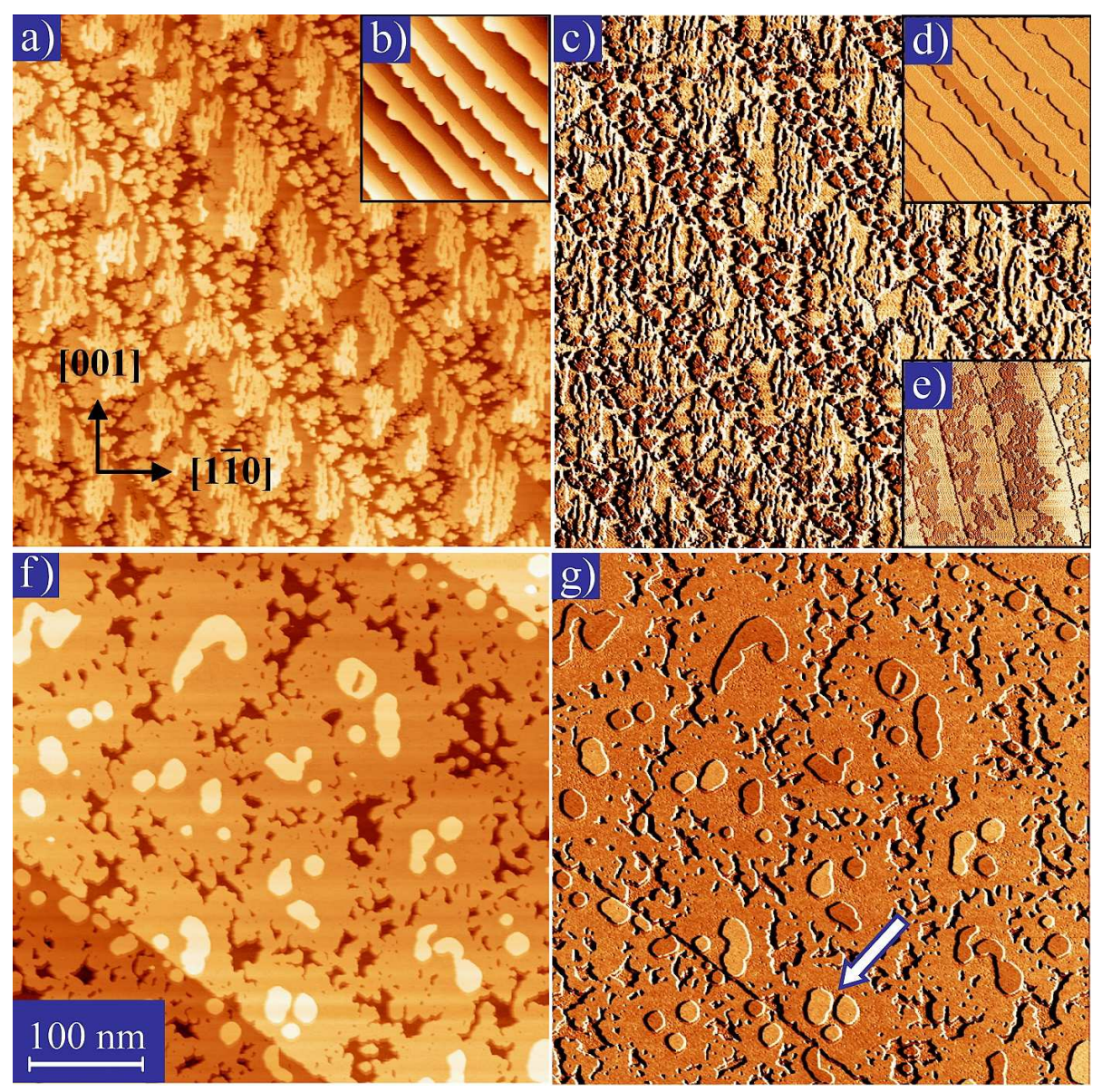

Fig. 1. Topographic STM images $\left(500 \times 500 \mathrm{~nm}^{2}\right)$ and simultaneously measured differential conductance $(\mathrm{d} I / \mathrm{d} U)$ maps for the following samples: (a) and (c) 0.5 ps-ML Fe grown on $\mathrm{Mo}(110)$ at $700 \mathrm{~K}$ and covered by $0.5 \mathrm{ML} \mathrm{Au}$ at RT. (b) and (d) images for the same sample as in (a) and (c), before Au coverage. (f) and (g) images for the same sample as in (a) and (c) taken after annealing at $700 \mathrm{~K}$. (e) $\mathrm{d} I / \mathrm{d} U$ map of 0.5 ML Au grown on a $\mathrm{Mo}(110)$ single crystal. Images and conductance maps were measured using the W/10 ML Au/4 ML Co magnetic tip with out-of-plane sensitivity, at the same applied voltage $(0.3 \mathrm{~V})$ and tunneling current $1.5 \mathrm{nA}$. Iron enriched FeAu nanostructures reveal an out-of-plane magnetic contrast. Direct lateral exchange coupling between adjacent islands is observed, as in the islands indicated by the arrow in $(\mathrm{g})$.

Due to different wetting conditions [5] (surface free energies: $\gamma_{\text {Mo }}=$ $2.9 \mathrm{~J} \mathrm{~m}^{-2}, \gamma_{\mathrm{Fe}}=2.9 \mathrm{~J} \mathrm{~m}^{-2}$, and $\left.\gamma_{\mathrm{Au}}=1.6 \mathrm{~J} \mathrm{~m}^{-2}\right)$ the morphology of the $\mathrm{Au}$ layer is different on the $\mathrm{Fe}(110) / \mathrm{Mo}(110)$ and on the clean $\mathrm{Mo}(110)$ surface. While on the clean Mo surface Au creates small islands (drops) coalescing in larger 
aggregates, on the surface of the Fe nanowires one can distinguish areas which are strongly elongated along [001], suggesting anisotropic diffusion of Au atoms on the $\mathrm{Fe}(110) / \mathrm{Mo}(110)$ surface. Anisotropic diffusion may be due to the two-fold symmetry of the bcc(110) surface. The thickness of the Au islands on Mo is $0.2 \mathrm{~nm}$ and in fair agreement with the bulk lattice atomic distance. The elongated areas on the ps-ML Fe are $0.37 \mathrm{~nm}$ higher than the pure Mo surface. The most probable assumption is that $\mathrm{Au}$ grows on the Fe monolayer. Mössbauer studies on $\mathrm{Au} / \mathrm{Fe} / \mathrm{W}(110)$ confirmed that $\mathrm{Fe}$ remains at the substrate surface even after $\mathrm{Au}$ coverage [17]. Alternatively, one can easily imagine that during the gold deposition the Fe atoms may be knocked out by Au atoms, leading to a homogeneous distribution of $\mathrm{Fe}$ and $\mathrm{Au}$ on Mo. This mixing does not take place, as we can conclude from the conductivity map presented in Fig. 1c. The Au islands reveal a different $\mathrm{d} I / \mathrm{d} U$ signal compared to the FeAu regions and uncovered Mo surface, similar to pure $\mathrm{Au}(0.5 \mathrm{ML})$ deposited on $\mathrm{Mo}(110)$. This element, specific $\mathrm{d} I / \mathrm{d} U$ contrast between pure $\mathrm{Au}$ and $\mathrm{Mo}(110)$, is easily visible on the conductivity map shown in Fig. 1e. Let us note that these spectroscopic images were measured at the same bias, which enables direct comparison of conductivities.

After annealing, the morphology of the sample is changed drastically. The Mo surface is almost completely covered by a homogeneous FeAu alloy revealing a homogeneous $\mathrm{d} I / \mathrm{d} U$ signal, as illustrated in Fig. If and g. There are also several round shaped islands (0.15 $\mathrm{nm}$ height) visible in the topographic STM image (Fig. 1f). They reveal different conductivities which are easily distinguished on the homogeneous $\mathrm{d} I / \mathrm{d} U$ background (Fig. $1 \mathrm{~g}$ ). It is very likely that these islands have the same composition, and thus, one can expect that they will reveal the same spin-averaged conductance signal. Therefore, we conclude that the observed $\mathrm{d} I / \mathrm{d} U$ contrast has a magnetic origin, similar to the case of clean Fe stripes, resulting from differences in the spin-dependent tunneling current between magnetic tip with an out-of-plane sensitivity and perpendicularly magnetized, presumably iron enriched, islands. Due to direct lateral exchange coupling, islands, which are very close to each other, reveal the same orientation of magnetization, as indicated by the arrow in Fig. 1g. The smallest island for which we can resolve an out-of-plane magnetic contrast has a diameter of $6 \mathrm{~nm}$. In this way we have shown that the size of a single bit can be diminished to objects consisting of a few hundred atoms [16].

\section{References}

[1] S. Heinze, M. Bode, A. Kubetzka, O. Pietzsch, X. Nie, S. Blügel, R. Wiesendanger, Science 288, 1805 (2000).

[2] W. Wulfhekel, J. Kirschner, Appl. Phys. Lett. 75, 1944 (1999).

[3] M. Bode, Rep. Prog. Phys. 66, 523 (2003).

[4] S.A. Wolf, D.D. Awschalom, R.A. Buhrman, J.M. Daughton, S. von Molnár, M.L. Roukes, A.Y. Chtchelkanova, D.M. Treger, Science 294, 1488 (2001).

[5] F.J. Himpsel, J.E. Ortega, G.J. Mankey, R.F. Willis, Adv. Phys. 47, 511 (1998). 
[6] A. Christensen, A.V. Ruban, P. Stoltze, K.W. Jacobsen, H.L. Skriver, J.K. Nørskov, F. Besenbacher, Phys. Rev. B 56, 5822 (1997).

[7] O.S. Hernán, A.L. Vázquez de Parga, J.M. Gallego, R. Miranda, Surf. Sci. 415, 106 (1998).

[8] N. Spiridis, J. Korecki, Surf. Sci. 507-510, 135 (2002).

[9] S.D. Bader, E.R. Moog, J. Appl. Phys. 61, 3729 (1987).

[10] F.J. Himpsel, Phys. Rev. B 44, 5966 (1991).

[11] K. Shintaku, Y. Daitoh, T. Shinjo, Phys. Rev. B 47, 14584 (1993).

[12] J. Unguris, R.J. Celotta, D.T. Pierce, J. Appl. Phys. 75, 6437 (1994).

[13] S.N. Okuno, K. Inomata, Phys. Rev. B 51, 6139 (1995).

[14] T. Duden, E. Bauer, in: Magnetic Ultrathin Films, Multilayers and Surfaces, Vol. 475, Mater. Res. Soc., Pittsburgh (PA USA) 1997, p. 283.

[15] J. Prokop, A. Kukunin, H.J. Elmers, Phys. Rev. B 73, 014428 (2006).

[16] M. Bode, O. Pietzsch, A. Kubetzka, R. Wiesendanger, Phys. Rev. Lett. 92, 067201 (2004).

[17] U. Gradmann, G. Liu, H.J. Elmers, M. Przybylski, Hyp. Interact. 57, 1845 (1990). 